
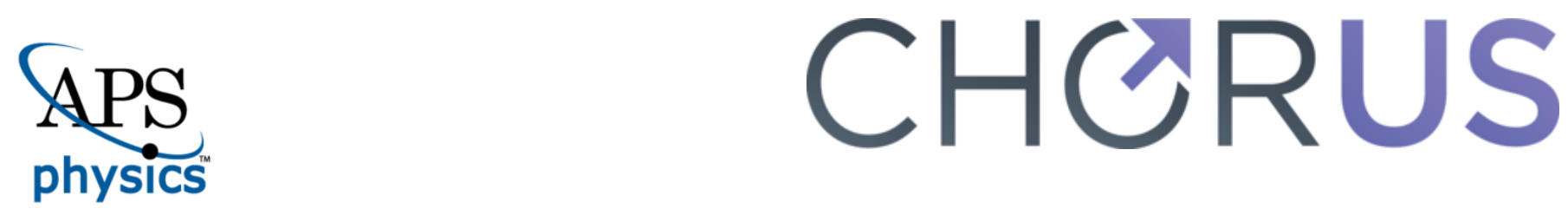

This is the accepted manuscript made available via CHORUS. The article has been published as:

\title{
Nonicosahedral boron allotrope synthesized at high pressure and high temperature
}

Irina Chuvashova, Elena Bykova, Maxim Bykov, Vitali Prakapenka, Konstantin Glazyrin, Mohamed Mezouar, Leonid Dubrovinsky, and Natalia Dubrovinskaia

Phys. Rev. B 95, 180102 - Published 16 May 2017

DOI: 10.1103/PhysRevB.95.180102 


\title{
First non-icosahedral boron allotrope synthesized at high pressure and high temperature
}

\author{
Irina Chuvashova ${ }^{\mathrm{a}, \mathrm{b}^{*}}$, Elena Bykova ${ }^{\mathrm{b}}$, Maxim Bykov ${ }^{\mathrm{b}}$, Vitali Prakapenka ${ }^{\mathrm{c}}$, Konstantin Glazyrin ${ }^{\mathrm{d}}$, \\ Mohamed Mezouar ${ }^{\mathrm{e}}$, Leonid Dubrovinsky ${ }^{\mathrm{b}}$, Natalia Dubrovinskaia ${ }^{\mathrm{a}}$ \\ ${ }^{a}$ Material Physics and Technology at Extreme Conditions, Laboratory of Crystallography, \\ University of Bayreuth, D-95440 Bayreuth, Germany \\ ${ }^{b}$ Bayerisches Geoinstitut, University of Bayreuth, D-95440 Bayreuth, Germany \\ ${ }^{c}$ Center for Advanced Radiation Sources, University of Chicago, 9700 South Cass Avenue, \\ Argonne, IL 60437, USA \\ ${ }^{d}$ Photon Science, Deutsches Elektronen-Synchrotron, Notkestrasse 85, D-22607 Hamburg, \\ Germany \\ ${ }^{e}$ European Synchrotron Radiation Facility, BP 220 F-38043 Grenoble Cedex, France \\ *Correspondence to: irina.chuvashova@gmail.com
}

\begin{abstract}
Theoretical predictions of pressure-induced phase transformations often become longstanding enigmas because of limitations of contemporary available experimental possibilities. Hitherto the existence of a non-icosahedral boron allotrope has been one of them. Here we report on the first non-icosahedral boron allotrope, which we denoted as $\zeta-\mathrm{B}$, with the orthorhombic $\alpha$ Ga-type structure (space group Cmce) synthesized in a diamond anvil cell at extreme highpressure high-temperature conditions $(115 \mathrm{GPa}$ and $2100 \mathrm{~K})$. The structure of $\zeta$-B was solved using single-crystal synchrotron X-ray diffraction and its compressional behavior was studied in the range of very high pressures $(115 \mathrm{GPa}$ to $135 \mathrm{GPa})$. Experimental validation of theoretical predictions reveals the degree of our up-to-date comprehension of condensed matter and promotes further development of solid state physics and chemistry.
\end{abstract}

Keywords: zeta-boron; gamma-boron; betta-boron; single-crystal X-ray diffraction; alfa-Ga structural type; high-pressure high-temperature phase transition.

\section{INTRODUCTION}

Boron has been widely studied due to its complex polymorphism (see the review article [1] and references therein). All five of the hitherto experimentally established boron allotropes ( $\alpha$-B, $\beta-\mathrm{B}, \gamma-\mathrm{B}, \delta$-B, and $\varepsilon-\mathrm{B})[1-6]$ belong to the family of icosahedral solids: their structures are based on various arrangements of $\mathrm{B}_{12}$ icosahedra, since three valence electrons of boron atoms are insufficient to form a simple covalent bond. However, von Schnering and Nesper [7] suggested 
the $\alpha$-Ga structure to be an alternative to the boron structure. According to these authors, the $\alpha$ Ga structure built up from open polyhedral fragments is strongly related to the closed polyhedral boron clusters $B_{n}$. Their extended Hückel calculations supported the $\alpha$-Ga model and showed that the $\alpha$-Ga structure is an appropriate covalent three-electron arrangement and is not electrondeficient [7].

The pressure-temperature (PT) experimental phase diagram of boron is currently limited to $20 \mathrm{GPa}$ and $3500 \mathrm{~K}[6,8-13]$, but there are a number of theoretical predictions concerning the behavior of boron at higher pressures and temperatures [14-21]. Häusserman et al. (2003) [15] predicted the phase transition from $\alpha$-B to $\alpha$-Ga-structured phase, accompanied by a nonmetalmetal transition at $74 \mathrm{GPa}$. The authors [15] used ab initio calculations employing pseudopotentials and a plane wave basis set in the framework of the density functional theory (DFT). The $\alpha$-Ga-structured boron phase was suggested to be stable up to $790 \mathrm{GPa}$; beyond this pressure a transition to the $f c c$ structure had to take place according to [15]. In parallel, Segal and Arias (2003) [22] performed calculations using a method based on perturbation theory and allelectron calculations with plane-wave-basis in DFT. The $\alpha$-Ga-structured boron was shown to be favorable in energy among other boron phases in the interval between 71 and $500 \mathrm{GPa}$. Note that at that time $\gamma$-B was not discovered yet. Later, a comparison of the stability of $\gamma$-B with respect to the $\alpha$-Ga-type boron phase led to consequent shift of their phase boundary from $78 \mathrm{GPa}$, as calculated in [14], to $89 \mathrm{GPa}$ [20], and $93 \mathrm{GPa}$ [19]. Calculations predict $\alpha$-Ga-structured boron to be an incompressible $\left(\mathrm{K}_{300}=263 \mathrm{GPa}\right)$ [18], superconducting material with strong anisotropy [23] due to its layered crystal structure [22,23]. Electric resistivity measurements [12] showed that compression of $\beta$-B leads to metallization of the material under investigation at pressures above $160 \mathrm{GPa}$ and ambient temperature, and to formation of a superconducting phase above $175 \mathrm{GPa}$ and $6 \mathrm{~K}$ [12]. The structure of metallic superconducting boron is unknown.

Although the idea of possible existence of boron with the $\alpha$-Ga structure is already more than 25 years old [7], it has remained difficult to prove. First, very high pressures are required for its synthesis, as predicted by [15]; second, boron is a weak X-ray scatterer that means that HPHT experiments are not straight forward and have to be done on a synchrotron; and third, a precursor material has to be of a very high purity, what is not always easy to reach with the highly reactive boron.

The logic of our experimental approach, aimed at overcoming the challenges listed above, is as follows. As a precursor material, we used single crystals of $\beta$ - $\mathrm{B}$ from the same synthesis batch as those which were fully characterized in our previous work [6]. The crystals were proven to be of high quality and high purity. To assure that we can follow the known $\beta$-B to $\gamma-\mathrm{B}$ transition at moderate pressures [4], at first we conducted a few synchrotron XRD experiments 
below one Mbar to see if our results are in accordance with our own previous observations and available literature data. Further experiments at higher pressures, up to above one Mbar, aimed at investigating the HP behavior of $\beta$-B to track possible phase transitions at room temperature (Eremets et al. [12] reported a visible step in the resistance of $\beta$-B at $110 \mathrm{GPa}$ ) and upon heating. Here, we report a new boron allotrope $(\zeta-\mathrm{B})$ with the $\alpha$-Ga-type structure synthesized from $\beta$-B at pressures over $115 \mathrm{GPa}$ and temperatures over $2100 \mathrm{~K}$ using a laser-heated diamond anvil cell (DAC). Its crystal structure was determined based on single-crystal synchrotron X-ray diffraction (XRD) data. The behavior of $\zeta$-B under compression from $115 \mathrm{GPa}$ to $135 \mathrm{GPa}$ was characterized using powder synchrotron XRD. 


\section{EXPERIMENTAL}

Synthesis of a precursor material. Single crystals of $\beta$-B studied in the present work at high pressures and high temperatures were synthesized using the high-pressure hightemperature technique described in detail in [6]. Their structure and purity were carefully characterized to assure the reliability of the obtained experimental results. The presence of impurities could be excluded.

Diamond-anvil cell experiments. The BX90-type diamond anvil cells (DAC) [24] made at Bayerisches Geoinstitut (Bayreuth, Germany) and Boehler-Almax type [25] beveled diamonds with the culet diameters of $120 \mu \mathrm{m}$ were used in high pressure experiments. Rhenium gaskets were squeezed between the anvils to make an indentation with the thickness of about $20 \mu \mathrm{m}$. Then in the center of the indentations, round holes of about $60 \mu \mathrm{m}$ in diameter were drilled. Two $\beta$-B crystals were placed into these chambers. Sizes of the crystals were about $10 \times$ $10 \times 15 \mu \mathrm{m}^{3}$ and orientation of the crystals was not specified. Neon was used as a pressure transmitting medium (PTM) and as pressure standard [26].

Single crystal synchrotron X-ray diffraction. Single crystals of $\beta$-B in a DAC were studied on ID27 at the European Synchrotron Radiation Facility (ESRF) and on P02.2 at PETRA III, DESY [27].

At ID27 diffraction data were collected at $293 \mathrm{~K}$ using the Perkin Elmer XRD1621 flat panel detector. The monochromatic radiation had the wavelength of $0.37380 \AA$ and the crystalto-detector distance was $383 \mathrm{~mm}$. Pressure in the cells was increased stepwise up to about $115 \mathrm{GPa}$, and single-crystal diffraction data for $\beta$-B were collected at each pressure point. 160 frames in the omega scanning range of $-40^{\circ}$ to $+40^{\circ}$ (in $0.5^{\circ}$ steps) were recorded with an exposure time of $2 \mathrm{~s}$. A portable double-sided laser heating system [28] was used to heat $\beta$-B crystals under pressure in experiments at ID27 (ESRF). Upon heating entire crystals were located in the laser beam and there were no measurable temperature gradients within the samples. The temperature variation during heating did not exceed $\pm 100 \mathrm{~K}$.

At P02.2 diffraction data were collected at $293 \mathrm{~K}$ using the Perkin Elmer XRD1621 detector. The monochromatic radiation had the wavelength of $0.29464 \AA$ and the crystal-todetector distance was $439 \mathrm{~mm}$. Data were collected at one pressure point at about $115 \mathrm{GPa}$ on the sample laser-heated at ID27. 152 frames in the omega scanning range of $-38^{\circ}$ to $+38^{\circ}$ were collected (in $0.5^{\circ}$ steps) with an exposure time of $10 \mathrm{~s}$ per frame.

Integration of the reflection intensities and absorption corrections were performed using CrysAlisPro software $[29,30]$. The structure of $\gamma$-B was refined in the anisotropic approximation for all atoms by full matrix least-squares. Due to small amount of the observed data the structure 
of $\zeta$-B was refined in isotropic approximation. Refinements of the crystal structures were performed using SHELXL software [31] implemented in the WinGX software package [30]. The crystallographic data of $\zeta$-B studied at $115 \mathrm{GPa}$ have been deposited in the Inorganic Crystal Structure Database [39]. The data may be obtained free of charge from Fachinformationszentrum Karlsruhe, 76344 Eggenstein-Leopoldshafen, Germany (Fax: +49 7247808 666; e-mail: crysdata@fiz-karlsruhe.de, http://www.fiz-karlsruhe.de/request_for_deposited_data.html) on quoting following CSD deposition number: 432572.

Powder XRD measurements. Samples of $\zeta$-B were studied at room temperature in angle-dispersive mode with a wavelength of $0.2952 \AA$ at the 13 -IDD beamline at APS, Argonne. Pressure in the cells was increased from 115 to $135 \mathrm{GPa}$ with a step of about $2 \mathrm{GPa}$. Diffraction images were collected at each pressure using a MAR CCD detector in the omega scanning range of $-20^{\circ}$ to $+20^{\circ}$ with an exposure time of $40 \mathrm{~s}$. The images were integrated using the DIOPTAS software [32] and the resulting diffraction patterns were processed using biased model in the EXPGUI software [33] in the GSAS Software package [34]. 


\section{RESULTS AND DISCUSSION}

In two independent DAC experiments at ID27 at ESRF single crystals of $\beta$-B were compressed up to 38(1) and 50(1) GPa, respectively. At these pressures the observed diffraction patterns perfectly match that of $\beta$-B (details of the HP structural studies of $\beta$-B will be published elsewhere). The lattice parameters and the molar volume of $\beta$-B corresponding to these two pressures are in agreement with the literature data of Sanz et al. [13], who measured the lattice parameters of $\beta$-B up to $100 \mathrm{GPa}$. Figure 1 shows the pressure dependence of the relative unit cell volume of $\beta-B$, as experimentally determined in [13] (black curve), and our two experimental points (black squares), which appear very close to the curve. After compression and the XRD measurements, the both crystals of $\beta$-B were double-sided laser heated to about $2000 \mathrm{~K}$. After the heating the pressure in the both DACs (determined using the equation of state of neon) increased and became 42(1) GPa in the first DAC and 58(1) GPa in the second one. In accordance with the experimental PT phase diagram of boron [6], a transition of $\beta$-B to $\gamma$-B took

place, and diffraction spots of $\gamma-\mathrm{B}$ were clearly observed in the diffraction pattern taken at room temperature (RT) after heating at 42 and $58 \mathrm{GPa}$. The quality of the HP single-crystal X-ray diffraction data was sufficient to refine both the lattice parameters and atomic coordinates of $\gamma-\mathrm{B}$ (details of the refinement of the structure of $\gamma-\mathrm{B}$ at 58(1) GPa are presented in Supplemental Material [35]). The structure of $\gamma$-B is orthorhombic (space group Pnnm) and built of covalently bonded $\mathrm{B}_{12}$ icosahedra and $\mathrm{B}_{2}$ dumbbells [36].

To compare the compressional behavior of various boron allotropes and to plot the P-V data on the same graph (Figure 1), the relative unit cell volumes for all considered allotropes were normalized to the unit cell volume of $\beta$-B per atom (320 atoms in $\beta$-B were accepted following [13]). The blue curve in Figure 1 corresponds to the experimental data for $\gamma$-B from [4]. Two experimental points obtained in the present study are shown by blue diamonds and lie on this curve.

In order to study the behavior of boron in a megabar pressure range, on ID27 at ESRF single crystals of $\beta$-B were first compressed up to 100(2) GPa. At this pressure at room temperature, all observed reflections were attributed to $\beta$-B. This point (the black square in Figure 1 corresponding to $100 \mathrm{GPa}$ ) fits well to the curve of Sanz et al. [13] extrapolated to $115 \mathrm{GPa}$ (dashed black curve). After double-sided laser-heating up to 2100(100) K using portable laser heating set up, pressure increased to 115(2) GPa. The material in the heated spot changed its color from dark-reddish to black (not reflecting). The X-ray diffraction pattern changed dramatically and had to be treated as of a powder sample. Apart from reflections of Re (gasket material) and Ne (used as PTM), several new relatively weak but clearly detectable 
reflections were observed. Their d-spacings perfectly matched those expected for $\alpha$-Ga-type structured boron, as predicted by Häusserman et al. [15]. The following orthorhombic lattice parameters were obtained at 115 GPa: $a=2.7159(11) \AA, b=4.8399(2) \AA, c=2.9565(6) \AA$.

The DAC with the sample described above was transported to the P02.2 beamline at PETRA III, where combination of a small size of the X-ray beam and its short wavelength increases chances for accumulating diffraction data suitable for single-crystal structural analysis. Detailed inspection of the diffraction pattern obtained on this beamline from the heated spot at 115 GPa (see Supplemental Material [35]) revealed individual single-crystal reflections related to the high-pressure high-temperature boron phase. Its structure was solved; some experimental details and crystallographic data are given in Supplemental Material [35]. The structure belongs to the $\alpha$-Ga structure type; it has the Cmce space group and 8 atoms per unit cell. The unit cell parameters determined from the single-crystal data $(a=2.7039(10) \AA, b=4.8703(32) \AA, c=$ 2.9697(6) $\AA$ ) are slightly different from those obtained from powder XRD at ID27, as they were determined using different sets of reflections, as well as different procedures of finding peaks positions, weight schemes in least square optimizations, etc. The new high-pressure boron allotrope with the $\alpha$-Ga-type structure was denoted as $\zeta-B$, sequentially after the fifth hitherto established boron allotrope, $\varepsilon$-B [5].

The structure of $\zeta-\mathrm{B}$ at 115(2) $\mathrm{GPa}$ is presented in Figure 2. It may be described as a stacking along the (010) direction of distorted and corrugated hexagonal nets (Figure 2A) with the $3^{6}$ topology, in accordance with the descriptions of von Schnering et al. [7,37] and Häusserman et al. [7,37]. Within every net each B atom connects to six neighbors, and the B-B bond lengths are 1.66(1) $\AA, 1.72(1) \AA$, and 1.75(1) $\AA$ (notated in Figure 2). The bonds between the nets bring the seventh neighbor to the coordination sphere of each boron atom (Figure $2 \mathrm{~B}$ ). These B-B bonds appear to be the shortest (1.59(1) $\AA$ ) in the structure of $\zeta$-B. Thus, despite seemingly 'layered' appearance, the $\zeta$-B structure is in fact a 3D network. Puckered fragments of the nets can be considered as open polyhedral fragments related to the closed icosahedral boron clusters.

As mentioned above, in $\zeta-\mathrm{B}$ each boron atom has a coordination number $(\mathrm{CN})$ equal to 7. In $\alpha$-B a half of boron atoms have $\mathrm{CN}=6$, and a half $\mathrm{CN}=7$ [38], and in $\gamma$ - $\mathrm{B}$ out of 28 atoms in the unit cell eight atoms have $\mathrm{CN}=6$, and other twenty atoms have $\mathrm{CN}=7$ [39]. For a complex and still controversial structure of $\beta$-B a simple count is not possible, but anyhow most of the boron atoms in this phase have $\mathrm{CN}=6$. Thus, comparing the structures of the boron allotropes, we observe a tendency to a rise of the $\mathrm{CN}$ of boron for high-pressure polymorphs. This agrees with the empiric rule that upon pressure-induced phase transition coordination number increases [40]. As expected [41], an average interatomic distance in the first coordination sphere is the longest 
for $\zeta$-B $(1.68 \AA)$ in comparison with corresponding values for $\alpha$-B (1.59 $\AA)$ [38], and $\gamma$-B (1.66 ̊) [39] at $115 \mathrm{GPa}$.

A phase transition of $\beta$-B to $\zeta-\mathrm{B}$ manifested in a drastic reduction of the molar volume: at $115 \mathrm{GPa}$ the molar volume of $\zeta$-B is by $\sim 7.5 \%$ less than that of $\beta$-B. According to theoretical calculations $[14,18,20]$ and our previous [6] and current experimental observations (up to about $60 \mathrm{GPa}), \gamma-\mathrm{B}$ is more stable at high pressures than $\beta$-B. If we compare the difference in the molar volume between $\gamma$-B and $\zeta$-B at $115 \mathrm{GPa}$, it is only about 3.1\% (see Figure 1, where the pressure dependence of the normalized volume of $\gamma$-B taken from [4] was plotted for the comparison).

It is worth mentioning here that we pressurized single crystals of $\beta$-B beyond the pressure $(110 \mathrm{GPa})$, at which Eremets et al. [12] observed a kink in the room-temperature $R(P)$ (resistance $v s$ pressure) curve. The authors [12] suggested the possibility that the transition of $\beta$-B to the metallic state occurs at $130 \mathrm{GPa}$. Häusserman et al. [15] proposed the $\alpha$-Ga structure as a structural model for a metallic high-pressure modification of $\mathrm{B}$ after a phase transition of either semiconducting icosahedral $\alpha-\mathrm{B}$ or $\beta$-B. We did not observe any transformations in $\beta$-B up to $115 \mathrm{GPa}$, and the transition to the $\alpha$-Ga-structured phase required heating to very high temperatures. Thus, it is very improbable that $\zeta-\mathrm{B}$ with the $\alpha$-Ga structure may be associated with the metallic high-pressure modification of boron discussed in $[12,15]$.

Further compression of the material synthesized at 115(2) GPa leads to decrease of the quality of single-crystal reflections. For this reason, the diffraction data of $\zeta$-B obtained up to $135 \mathrm{GPa}$ on 13-IDD at the APS were integrated to 1D '2-theta' scans. An example of a diffraction pattern at 121(2) GPa is presented in Figure 3. The unit cell parameters decreased with pressure. The linear compressibility along the $a$ and $c$ axes was found to be $\sim 7(3)^{*} 10^{-4} \mathrm{GPa}^{-}$ ${ }^{1}$, and along the $b$ axis - a bit lower, $\sim 4(3)^{*} 10^{-4} \mathrm{GPa}^{-1}$ that can be explained by the shortest B-B bonds in the (010) direction. The PV data set of $\zeta$-B in the pressure range of 115 to $135 \mathrm{GPa}$ was fitted using the second-order Birch-Murnaghan (2BM) equation of state (EoS) and gave the following EoS parameters: $V_{115}=39.20(8) \AA^{3}$ and $\mathrm{K}_{115}=575(65) \mathrm{GPa} ; \mathrm{K}^{\prime}=4$ (fixed) $\left(V_{115}\right.$ is the unit cell volume and $K_{115}$ is the bulk modulus at $115 \mathrm{GPa}$ and room temperature; $K^{\prime}$ is the pressure derivative of the bulk modulus) (Table I). Use of the Vinet EoS led to the following parameters: $\mathrm{V}_{115}=39.19(8) \AA^{3}$ and $\mathrm{K}_{115}=577(65) \mathrm{GPa} ; \mathrm{K}^{\prime}=4$ (fixed).

The bulk moduli of $\alpha$-Ga-structured boron at ambient conditions were calculated in $[18,19,23]$. To compare these predictions with our experimental results, we computed the theoretical values of the bulk moduli to be at $115 \mathrm{GPa}$ according to these three papers (Table I). The EoSFit-7c software [42] was used. As seen, experimental values of the bulk modulus are lower than the theoretically predicted ones. The pressure dependence of the unit cell volume of $\zeta$-B, as experimentally determined in the present work and theoretically predicted $[18,19,23]$ is 
presented in Supplemental Material [35]. There is a significant difference (about 4\%) between the earlier [23] and recent [18,19] theoretical predictions. Our experimental results are in reasonable agreement with data of [18,19] (see Supplemental Material [35]). 


\section{CONCLUSIONS}

To verify theoretical predictions regarding the existence of $\alpha$-Ga-structured boron and its behavior at high pressures $[7,14,15,19,20]$, we have conducted a series of high-pressure hightemperature experiments. We demonstrated that the predicted boron allotrope [7,15] can be obtained by laser-heating of single crystals of $\beta$-B to over $2100 \mathrm{~K}$ at pressures above $115 \mathrm{GPa}$. This phase, which we call $\zeta$-B, has the $\alpha$-Ga-type orthorhombic structure as revealed by singlecrystal X-ray diffraction. Measured precisely interatomic distances and linear compressibilities along the major crystallographic directions do not allow interpreting the structure as layered, as earlier proposed [23]. In the studied pressure range (from 115 to $132 \mathrm{GPa}$ ) $\zeta$-B is less compressible than any other boron allotropes known so far. Based on our experimental data we do not see a relation between $\zeta$-B and the metallic high-pressure modification of $\mathrm{B}$ discussed by $[12,15]$.

\section{ACKNOWLEDGEMENTS}

We thank G. Parakhonskiy for providing us with samples of $\beta$-B used here. N.D. thanks the German Research Foundation (Deutsche Forschungsgemeinschaft (DFG)) and the Federal Ministry of Education and Research (BMBF; Germany) for financial support through the DFG Heisenberg Programme (projects no. DU 954-6/1 and DU 954-6/2) and project no. DU 954-8/1 and the BMBF grant no. 5K13WC3. L.D. thanks the DFG and the BMBF (Germany) for financial support. 


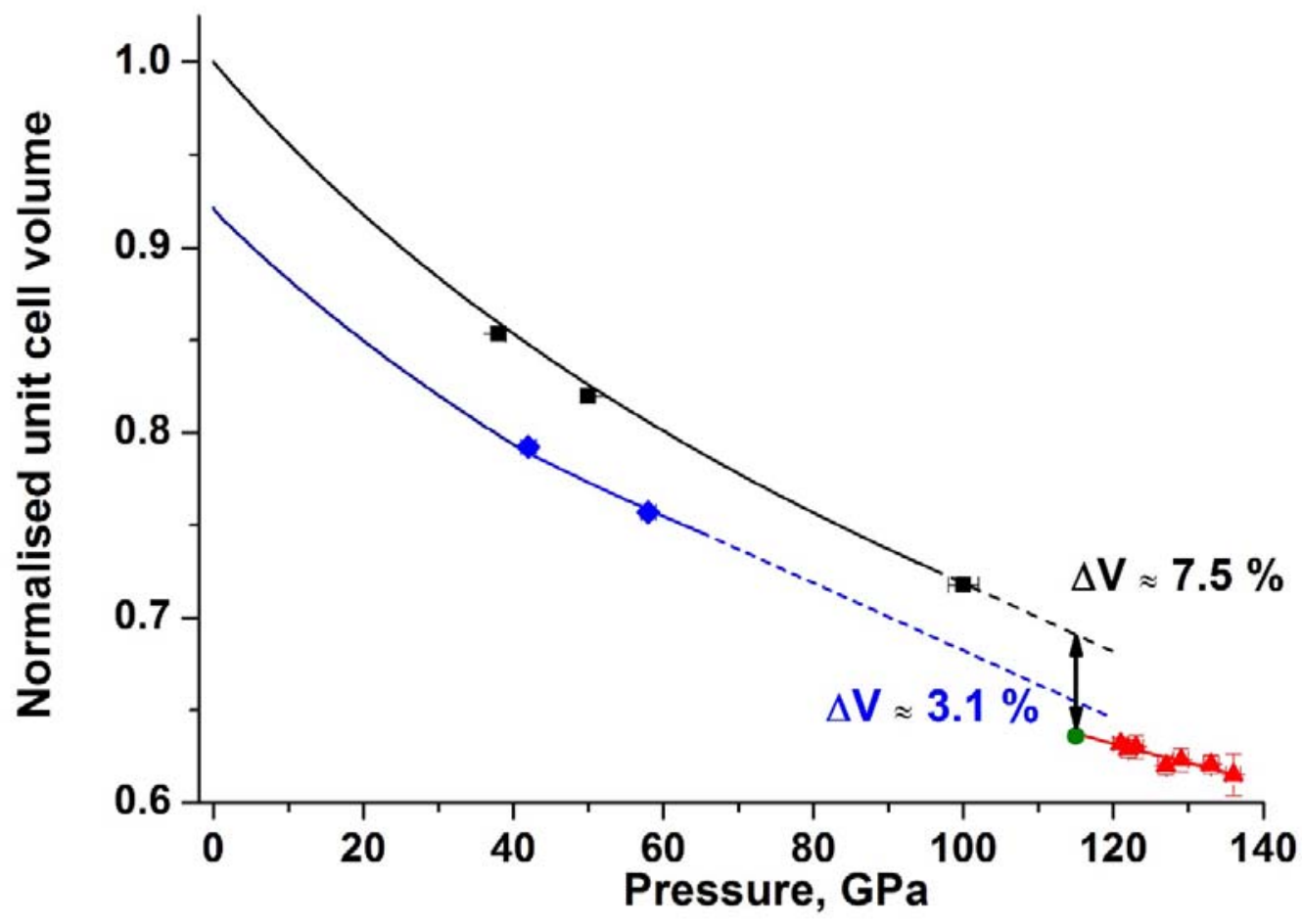

FIG. 1. (Color online) The pressure dependence of the normalized relative unit cell volumes of three boron allotropes, $\beta$-B, $\gamma$-B and $\zeta$-B. The volumes were normalized on the volume of $\beta$-B per atom calculated from the experimental data of Sanz et al. [13]. The solid black curve corresponds to the Vinet equation of state of $\beta$-B up to $100 \mathrm{GPa}$ from [13] $\left(\mathrm{V}_{0}=2460 \AA^{3}, \mathrm{~K}_{300}=210(6) \mathrm{GPa}, \mathrm{K}^{\prime}=2.23\right)$. Dashed black curve is its extrapolation to $120 \mathrm{GPa}$. Black squares represent our experimental data points for $\beta$-B (see text). The green circle represents the volume data for $\zeta$-B obtained from our single-crystal XRD at 115 GPa (after laser-heating of $\beta$-B at this pressure a phase transition occurred, accompanied with the volume reduction by ca.7.5\%). The red triangles correspond to the PV data of $\zeta$-B obtained from powder XRD. Their fit with the 2BM EoS is shown by the red solid curve. The blue curve corresponds to the 3BM EoS of $\gamma$-B according to [39] (below 40 GPa: $\mathrm{V}_{0}=198.1(3) \AA^{3}, \mathrm{~K}_{300}=227(3) \mathrm{GPa}, \mathrm{K}^{\prime}=2.5(2)$; above $45 \mathrm{GPa}: \mathrm{V}_{0}=192.6(3) \AA^{3}, \mathrm{~K}_{300}=281(6) \mathrm{GPa}, \mathrm{K}^{\prime}=2.8(9)$ ). Its extrapolation to $120 \mathrm{GPa}$ is shown by a dashed blue curve. Blue diamonds show our two experimental points (see text). The volume difference between $\gamma$-B and $\zeta$-B at $115 \mathrm{GPa}$ is ca. $3.1 \%$. 

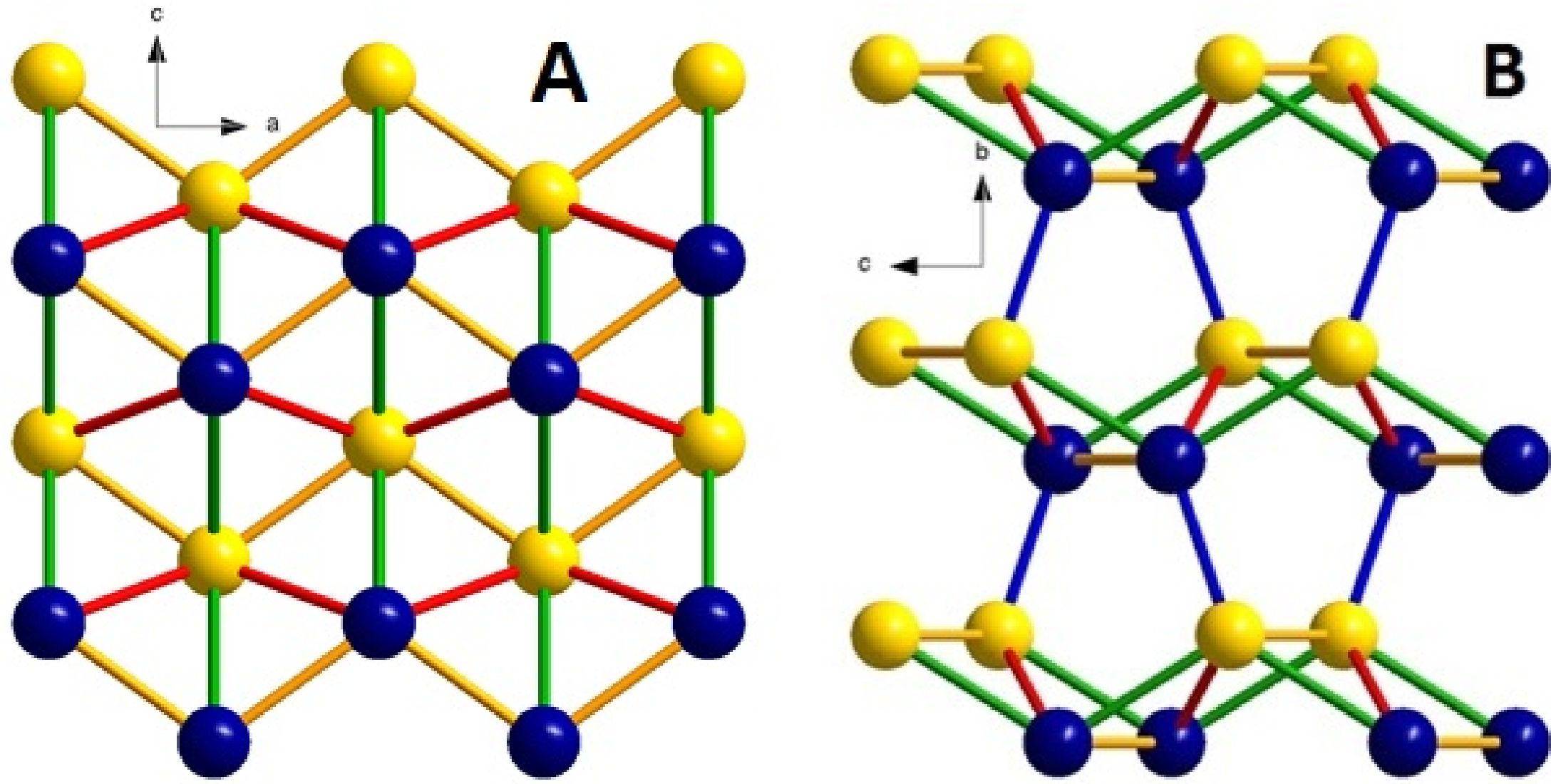

FIG. 2. (Color online) The structure of $\zeta-$ B. A. The projection of a fragment of distorted and corrugated hexagonal nets on the $a c$ plane. Such nets are stacked along the (010) direction. Blue and yellow atoms do not lie in the same plain (blue atoms are lower and yellow ones are upper if seen along the (010) direction). Bonds with different lengths are shown in different colors: 1.66(1) $\AA$ (orange), 1.72(1) $\AA$ (red), and 1.75(1) $\AA$ (green). B. The projection of three nets on the $b c$ plain. The length of bonds connecting the layers is $1.59(1) \AA$ (blue). 


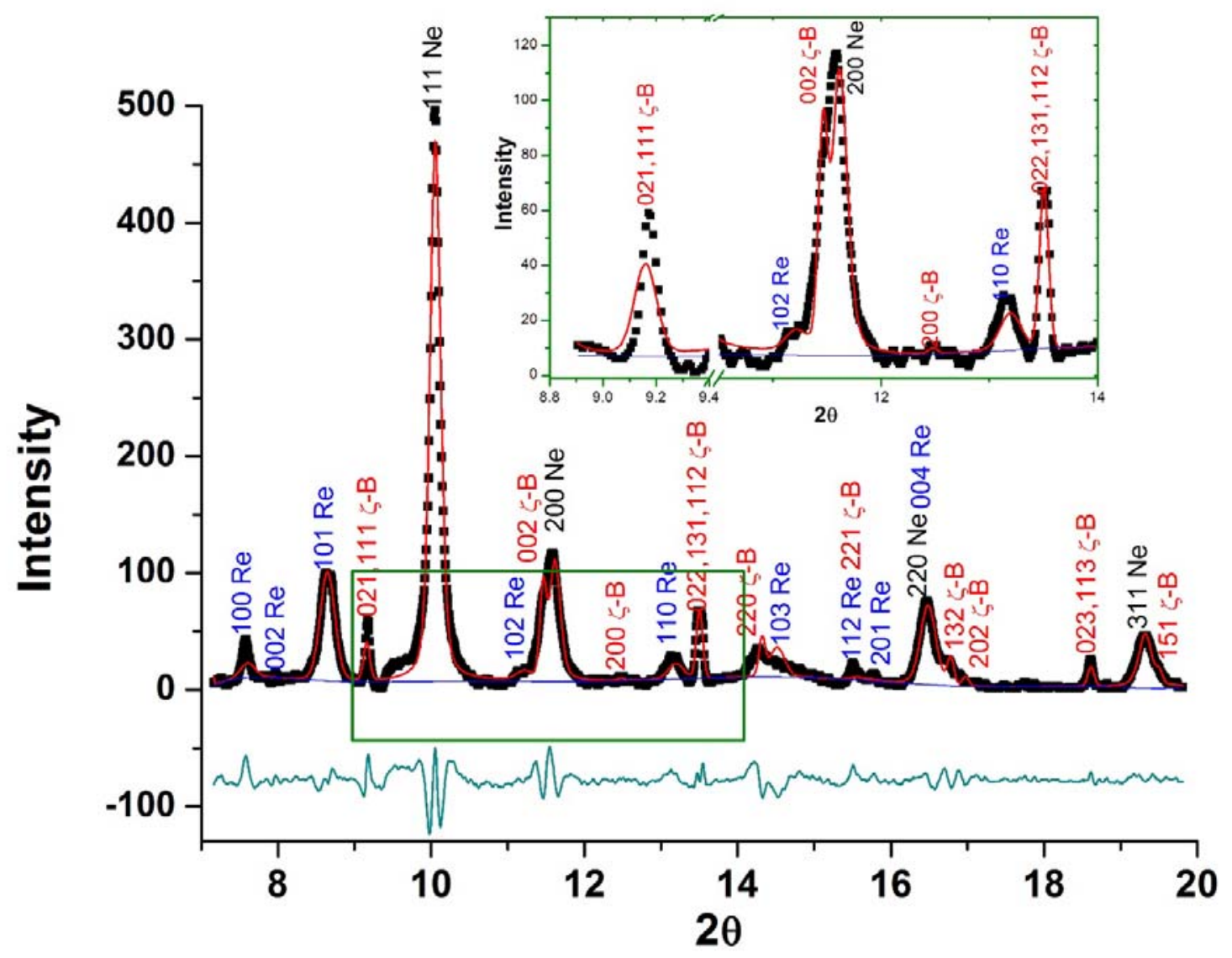

FIG. 3. (Color online) X-ray diffraction pattern of $\zeta$-B obtained at 121(2) GPa. Black dots represent experimental data. Red solid line derives the refinement using biased model, blue solid line states for calculations of background. Green curve is the intensity difference $\left(I_{\text {obs }}-I_{\text {calc }}\right)$ between experimental data and calculations. Reduced $\chi^{2}=1.953$. The reflections are assigned to $\mathrm{Re}, \mathrm{Ne}$, and $\zeta$-B; their $h k l$ are designated in different colors for clarity. 
TABLE I. Parameters of the equations of state of $\zeta$-B compared to theoretical predictions. The EoSes are designated as follows: $\mathrm{BM}$ stays for the Birch-Murnaghan (3BM for the $3^{\text {rd }}$ order, $2 \mathrm{BM}$ for the $2^{\text {nd }}$ order), $\mathrm{M}$ for Murnaghan and V for Vinet.

\begin{tabular}{cccccc}
\hline \hline \multicolumn{1}{c}{$\zeta-\mathbf{B}$} & $\mathbf{E o S}$ & $\mathbf{V}_{\mathbf{1 1 5}}, \AA^{\mathbf{3}}$ & $\mathbf{K}_{\mathbf{1 1 5}}, \mathbf{G P a}$ & $\mathbf{K}^{\prime}$ & Ref. \\
\hline Experiment & $\mathrm{V}$ & $39.19(8)$ & $577(65)$ & 4 & present study \\
ab initio & 2BM & $39.20(8)$ & $575(66)$ & 4 & present study \\
calculations & 2BM & $38.99(4)$ & 626 & 3.6 & {$[19]$} \\
& $\mathrm{BM}$ & $39.11(4)$ & 674 & 4 & {$[18]$} \\
& $\mathrm{M}$ & $38.09(3)$ & 640 & 3.26 & {$[23]$} \\
\hline \hline
\end{tabular}




\section{REFERENCES}

[1] B. Albert and H. Hillebrecht, Angew. Chem. Int. Ed. 48, 8640 (2009).

[2] G. Parakhonskiy, N. Dubrovinskaia, L. Dubrovinsky, S. Mondal, and S. van Smaalen, Journal of Crystal Growth 321, 162 (2011).

[3] R. J. Nelmes, J. S. Loveday, D. R. Allan, J. M. Besson, G. Hamel, P. Grima, and S. Hull, Phys. Rev. B 47, 7668 (1993).

[4] E. Y. Zarechnaya et al., Phys. Rev. Lett. 102, 185501, 185501 (2009).

[5] G. Parakhonskiy, N. Dubrovinskaia, E. Bykova, R. Wirth, and L. Dubrovinsky, High Press. Res. 33, 673 (2013).

[6] G. Parakhonskiy, N. Dubrovinskaia, E. Bykova, R. Wirth, and L. Dubrovinsky, Sci. Rep. 1, 96, 96 (2011).

[7] H. G. von Schnering and R. Nesper, Acta Chemica Scandinavica 45, 870 (1991).

[8] J. Qin, T. Irifune, H. Dekura, H. Ohfuji, N. Nishiyama, L. Lei, and T. Shinmei, Phys. Rev. B 85, 014107 (2012).

[9] Y. Z. Ma, C. T. Prewitt, G. T. Zou, H. K. Mao, and R. J. Hemley, Phys. Rev. B 67, $174116,174116(2003)$.

[10] R. H. Wentorf, in Boron: Volume 2: Preparation, properties, and applications, edited by G. K. Gaulé (Springer US, Boston, MA, 1965), pp. 97.

[11] M. Kaneshige, S. Hirayama, T. Yabuuchi, T. Matsuoka, K. Shimizu, Y. Mita, H. Hyoudo, and K. Kimura, Journal of the Physical Society of Japan 76, 19 (2007).

[12] M. I. Eremets, V. V. Struzhkin, H.-k. Mao, and R. J. Hemley, Science 293, 272 (2001).

[13] D. N. Sanz, P. Loubeyre, and M. Mezouar, Phys. Rev. Lett. 89, 245501, 245501 (2002).

[14] S. Aydin and M. Simsek, J. Alloys Compd. 509, 5219 (2011).

[15] U. Haussermann, S. I. Simak, R. Ahuja, and B. Johansson, Phys. Rev. Lett. 90, 4, 065701 (2003).

[16] Y. Zhao, Q. Xu, L. J. Simpson, and A. C. Dillon, Chemical Physics Letters 496, 280 (2010).

[17] A. R. Oganov and V. L. Solozhenko, Journal of Superhard Materials 31, 285 (2009).

[18] Y.-H. Xu, H.-Y. Liu, X.-F. Hao, R.-N. Chen, and F.-M. Gao, Chinese Physics Letters 32, 026101 (2015).

[19] C. Fan, J. Li, and L. Wang, Sci. Rep. 4, 6786 (2014).

[20] A. R. Oganov et al., Nature 457, 863 (2009).

[21] D. Li, K. Bao, F. Tian, X. Jin, D. Duan, Z. He, B. Liu, and T. Cui, RSC Advances 4, 203 (2014).

[22] D. E. Segall and T. A. Arias, Phys. Rev. B 67, 064105 (2003).

[23] Y. Ma, J. S. Tse, D. D. Klug, and R. Ahuja, Phys. Rev. B 70, 214107 (2004).

[24] I. Kantor, V. Prakapenka, A. Kantor, P. Dera, A. Kurnosov, S. Sinogeikin, N. Dubrovinskaia, and L. Dubrovinsky, Rev. Sci. Instrum. 83, 6, 125102 (2012).

[25] R. Boehler and K. De Hantsetters, High Press. Res. 24, 391 (2004).

[26] Pressure scale from EOS.

[27] H.-P. Liermann et al., Journal of Synchrotron Radiation 22, 908 (2015).

[28] I. Kupenko et al., Rev. Sci. Instrum. 83, 124501 (2012).

[29] A. Technologies, A. Technologies, and O. UK Ltd., UK, CrysAlisPro Software system, CrysAlisPro Software system, 2013.

[30] L. J. Farrugia, J. Appl. Crystallogr. 45, 849 (2012).

[31] G. M. Sheldrick, Acta Crystallographica Section A 64, 112 (2008).

[32] C. Prescher and V. B. Prakapenka, High Press. Res. 35, 223 (2015).

[33] B. Toby, J. Appl. Crystallogr. 34, 210 (2001).

[34] A. C. Larson and R. B. Von Dreele, Los Alamos National Laboratory Report LAUR 86$748(2000)$. 
[35] See Supplemental Material at [URL] for details of the refinement of structures of $\gamma$-B and $\zeta$-B, diffraction pattern at $115 \mathrm{GPa}$ and pressure dependence of the unit cell volume of $\zeta-\mathrm{B}$.

[36] E. Y. Zarechnaya, L. Dubrovinsky, N. Dubrovinskaia, N. Miyajima, Y. Filinchuk, D. Chernyshov, and V. Dmitriev, Sci. Technol. Adv. Mat. 9, 4, 044209 (2008).

[37] U. Häussermann, S. Lidin, S. I. Simak, and I. A. Abrikosov, Chemistry - A European Journal 3, 904 (1997).

[38] I. Chuvashova, E. Bykova, M. Bykov, V. Svitlyk, B. Gasharova, Y.-L. Mathis, R. Caracas, L. Dubrovinsky, and N. Dubrovinskaia, J. Solid State Chem. 245, 50 (2017).

[39] E. Zarechnaya, N. Dubrovinskaia, R. Caracas, M. Merlini, M. Hanfland, Y. Filinchuk, D. Chernyshov, V. Dmitriev, and L. Dubrovinsky, Phys. Rev. B 82, 184111, 184111 (2010).

[40] A. Neuhaus, Chimia 18, 93 (1964).

[41] W. Kleber, Kristall und Technik 2, 13 (1967).

[42] J. Angel Ross, M. Alvaro, and J. Gonzalez-Platas, in Zeitschrift für Kristallographie Crystalline Materials 2014), p. 405. 